\title{
Fibrin sealants in lumbar annuloplasty after endoscopic discectomy as a method to prevent recurrent lumbar disc herniation
}

\author{
Pooya Torkian (1), Keivan Daneshvar (2), Ebrahim Taherian (3), Yasaman Rezaeifar \\ (4), Shahram Akhlaghpoor (3) \\ (1) School of Medicine, Shahid Beheshti University of Medical Sciences, Tehran, Iran; (2) \\ Department of Diagnostic, Interventional and Pediatric Radiology, Inselspital, Bern University \\ Hospital, University of Bern, Bern, Switzerland; (3) ) Pardis Noor Medical Imaging Center, \\ Tehran, Iran; (4) Department of Psychology, Faculty of Health, York University, Toronto, \\ Ontario, Canada. \\ This article is distributed under the terms of the Creative Commons Attribution Noncommercial License (CC BY-NC 4.0) which permits \\ any noncommercial use, distribution, and reproduction in any medium, provided the original author(s) and source are credited.
}

\begin{abstract}
Fibrin sealant as a promising agent for providing scaffold and efficient hemostasis is widely accepted in several specialties. However, the outcome of Fibrin sealants in lumbar annuloplasty after endoscopic discectomy has not been evaluated in patients with disc herniation. The goal of this study was to evaluate the efficacy, response, and probability of future recurrence rates in herniated nucleus pulposus (HNP) with the use of fibrin sealant in conjunction with endoscopic disc surgery. A total of 35 patients ( $28 \mathrm{men}, 7$ women) were evaluated, including 18 patients who underwent endoscopic discectomy alone and 17 patients that received fibrin sealant at the site of annulus tear and endoscopic discectomy. All patients were followed through both clinical and imaging methods for an average of 10.5 months. Primary outcome measure was defined as lumbar decompression approved by imaging and symptom alleviation after endoscopic spinal discectomy with Visual Analogue Scale (VAS) score $\leq 4$ (cut-off point). Median size of annular tearing was significantly lower in the endoscopic discectomy group (median, 3) (minimum, 2; maximum, 5); however, the corresponding factor in the endoscopic discectomy plus fibrin sealant group was significantly larger (median, 6) (minimum, 5; maximum, 10), with $P<0.001$. Only one patient in the endoscopic discectomy group had an HNP recurrence during follow-up compared to two patients in the endoscopic discectomy plus fibrin sealant group. Due to the temporary effects of fibrin sealant in preventing disc herniation and the observed recurrence rate in both the case and control groups, the results of this study suggest a role of fibrin sealants combined with endoscopic discectomy to prevent early HNP recurrence rate.
\end{abstract}

Key Words: Fibrin sealant, endoscopic discectomy, disc herniation.

Eur J Transl Myol 30 (2): 325-333, 2020

Low back pain (LBP) is a pervasive problem, estimated to affect up to $80 \%$ of people during their life. ${ }^{1,2}$

Disc herniation as a consequence of annulus rupture can be accelerated by obesity, overuses injuries, trauma, straining of the spine, and strenuous activity. Tearing in annulus fibrosis results in herniation of nucleus pulposus (HNP) through fibers in intervertebral or vertebral foramen and pressure on a nerve root (pinched nerve). ${ }^{1}$ Larger disc herniation and patients who are unresponsive to medical management can be a candidate for fenestration, laminectomy with or without fixation, minimally invasive percutaneous therapy, annuloplasty, or endoscopic discectomy. ${ }^{3,4}$ Hitherto, some studies have shown that after fenestration surgical procedures, reoperation rates can occur based on the level of lumbar disc herniation and patient characteristics. ${ }^{5-7}$ As a type of novel tissue-persevering disc surgery, endoscopic discectomy bears a resemblance to lumbar disc fenestration. Formerly, fibrin sealants were used in neurosurgery to suture line closures and prevent cerebrospinal fluid leaks. ${ }^{8}$ In this study, we introduce and describe a new surgical technique, which involves injecting fibrin sealants in lumbar annuloplasty site after endoscopic discectomy. The scope of this study was to evaluate the efficacy, response, and probability of future recurrence rates in HNP with the use of fibrin sealant in conjunction with endoscopic disc surgery, in comparison to endoscopic lumbar discectomy. 


\section{Materials and Methods}

\section{Enrollment of patients}

In this prospective study from August 2014 through December 2017, we enrolled 35 patients (28 men, 7 women) in Pardis Noor medical center. Endoscopic discectomy was performed on all patients by an interventional radiologist, accompanied by an orthopedic surgeon with 10 years of experience in spinal surgery. All procedures were done by endoscopic spine system (Richard Wolf GmbH, Knittlingen, Germany). The selected procedure in the study and the control group were the same, with the exception of injecting extra fibrin sealants in the study group. After generating a good view of the operative field, we created a grading scale based on the size of annular tearing (Figure 1) to select patients who could receive sealants. They were grouped as follows: grade 1: 0-2 mm; grade 2: 2-5 mm; and grade 3: more than $5 \mathrm{~mm}$. Patients in grade 3 were considered the best candidates for injecting fibrin sealants. In control group (18 patients), only endoscopic discectomy was performed and in the study group (17 patients) fibrin sealants were injected in lumbar annuloplasty site after endoscopic discectomy. Informed consent forms with detailed possible risks and benefits of this new technique were signed by all patients after a counseling session. Our research adhered to the Declaration of Helsinki and regulations of the Health Insurance Portability and Accountability Act. Patients with LBP were included in our study after meeting the selection criteria: 1) inability to do routine activity; 2) disc protrusion/extrusion with uni/bilateral radiculopathy; 3) no response to conservative managements, including spinal orthosis, bed rest, anti-inflammatory agents, analgesics, and

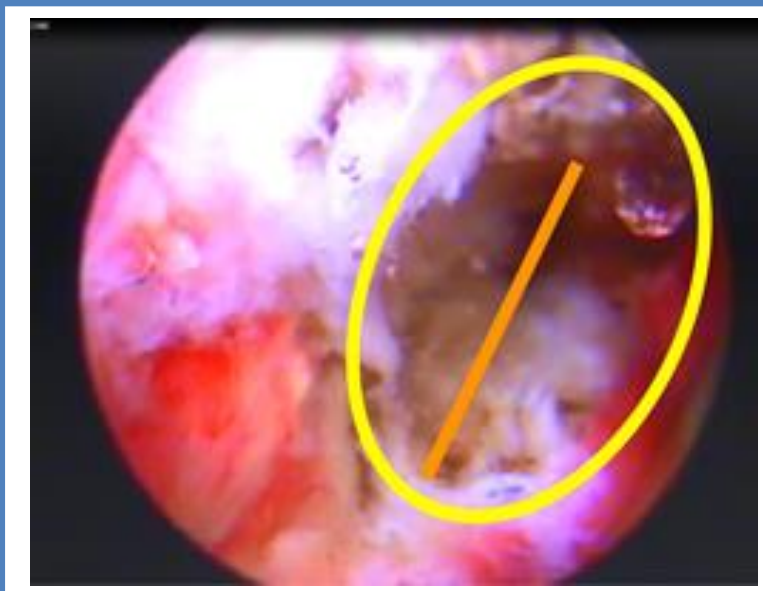

Fig 1. Grading scale based on the size of annular tearing. These classifications were as follows: grade 1: 0-2 mm; grade 2: 2-5 mm; and grade 3: more than $5 \mathrm{~mm}$. Yellow circle depicts the whole suspected area of tearing. The orange line shows the edge-to-edge measurement of tearing muscle relaxants with lumbar stabilization; and 4) positive straight leg raise with evidence of nerve involvement, radiculopathy, and muscle atrophy.

Exclusion criteria were as follows: patients who had 1) a previous surgery at the same level of the herniated disc; 2) symptoms or signs of systemic infection or localized infection at the expected needle entry sites; 3 ) history of coagulopathy or use of anti-platelets or thrombolytics in the previous 2 weeks; and 4) recurrent disc herniation with adhesions at the same level of the herniated disc. Before the operation, complete blood tests and lumbar radiography or magnetic resonance imaging (MRI) in anteroposterior, axial and lateral views were obtained from all patients. Our radiologists who were blinded to the study design, made a diagnosis of lumbar disc herniation. Collected data included age at operation, sex, duration of symptoms, location of pain, type of lumbar disc herniation, level of lumbar involvement, preoperative history, and physical examination, including straight leg raise, patella and achilles reflexes, and calf circumference measurement. To measure the pre- and post-operative clinical status and functional status of patients, Visual Analogue Scale (VAS) results were recorded (VAS: $0=$ no pain, VAS: $10=$ intolerable pain). During follow-ups, reflexes were graded at the bedside in a three-step manner (intact-impairedalleviated) compared to their prior responses. Patients were early mobilized after operation to reducing rate of complications and morbidity. ${ }^{9}$

Post-operative follow-ups were done regularly based on their scheduled appointments, initially 1 month after surgery and then every 3-6 months, by performing clinical examinations. MRI in anteroposterior, axial and lateral views were obtained up to 2 days and 6 months

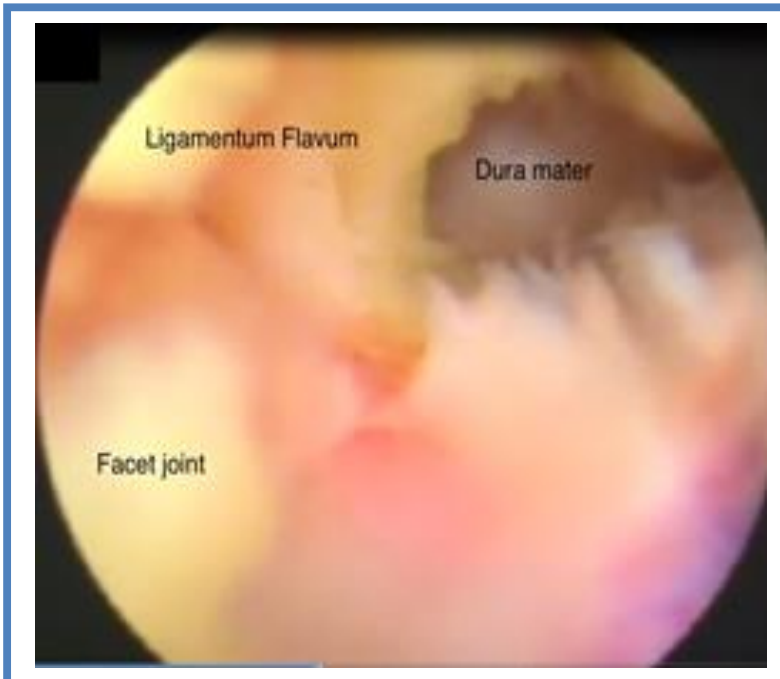

Fig 2. Endoscopic visualization of Percutaneous Interlaminar Lumbar Discectomy by punching Ligamentum Flavum with micro punch (Ø $2.5 \mathrm{~mm}$, WL $360 \mathrm{~mm}$ ) to afford a good view of dura mater 


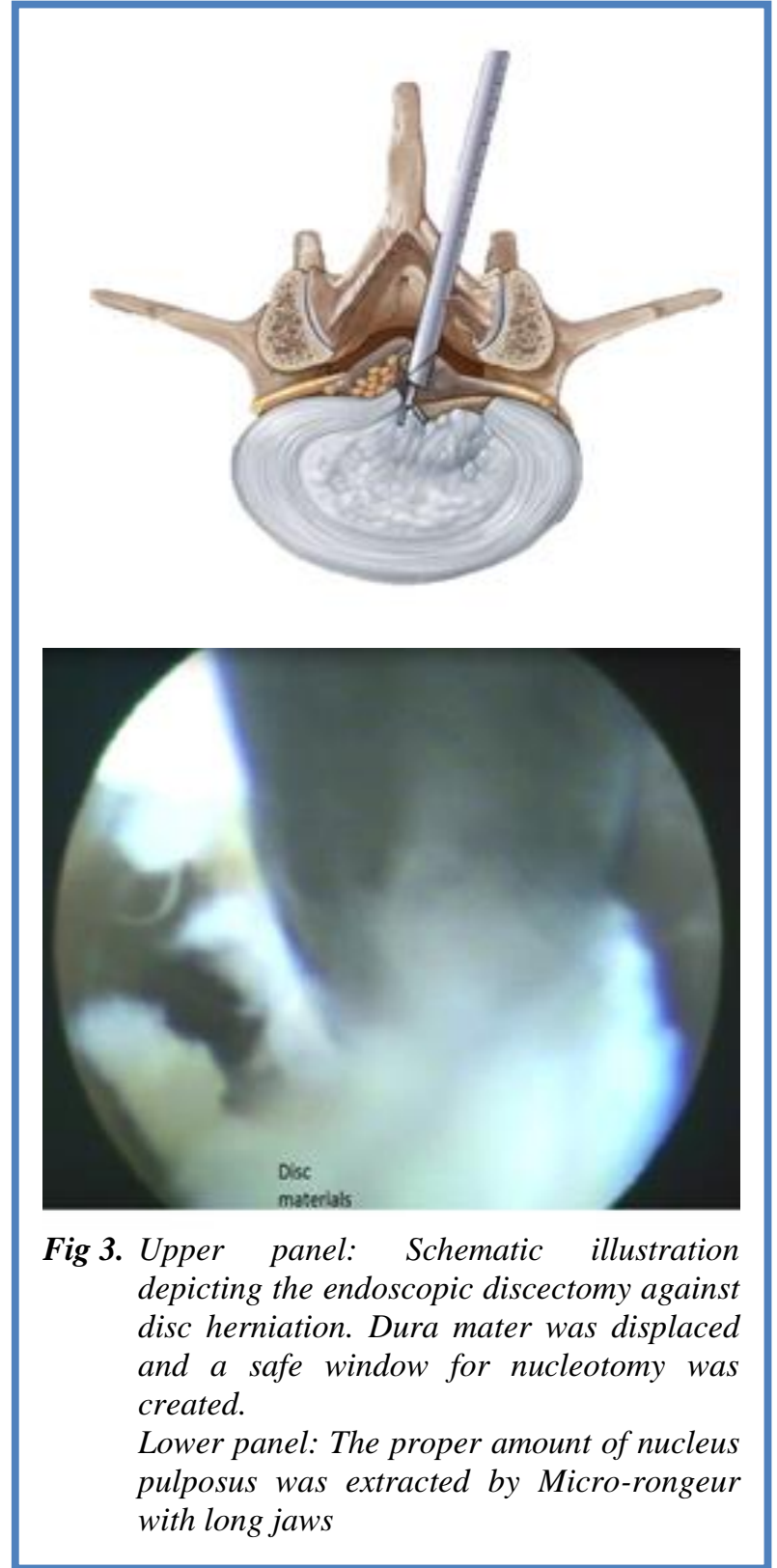

after an operation. The average post-operative follow-up was 10.5 months (minimum: 6 months, maximum: 24 month). Recurrent lumbar disc herniation was diagnosed in our center during follow-up based on clinical presentation and on returning similar prior symptoms in the ipsilateral or contralateral side after the index operation. Primary outcome measure was defined as lumbar decompression approved by imaging and symptom alleviation after endoscopic spinal discectomy with VAS score $\leq 4$ (cut-off point) compare to preoperative VAS score.

Surgical Technique: Percutaneous Interlaminar Lumbar Discectomy

We used the endoscopic spine system (Richard Wolf $\mathrm{GmbH}$, Knittlingen, Germany). The goal of this surgical technique was to remove herniated disc material through

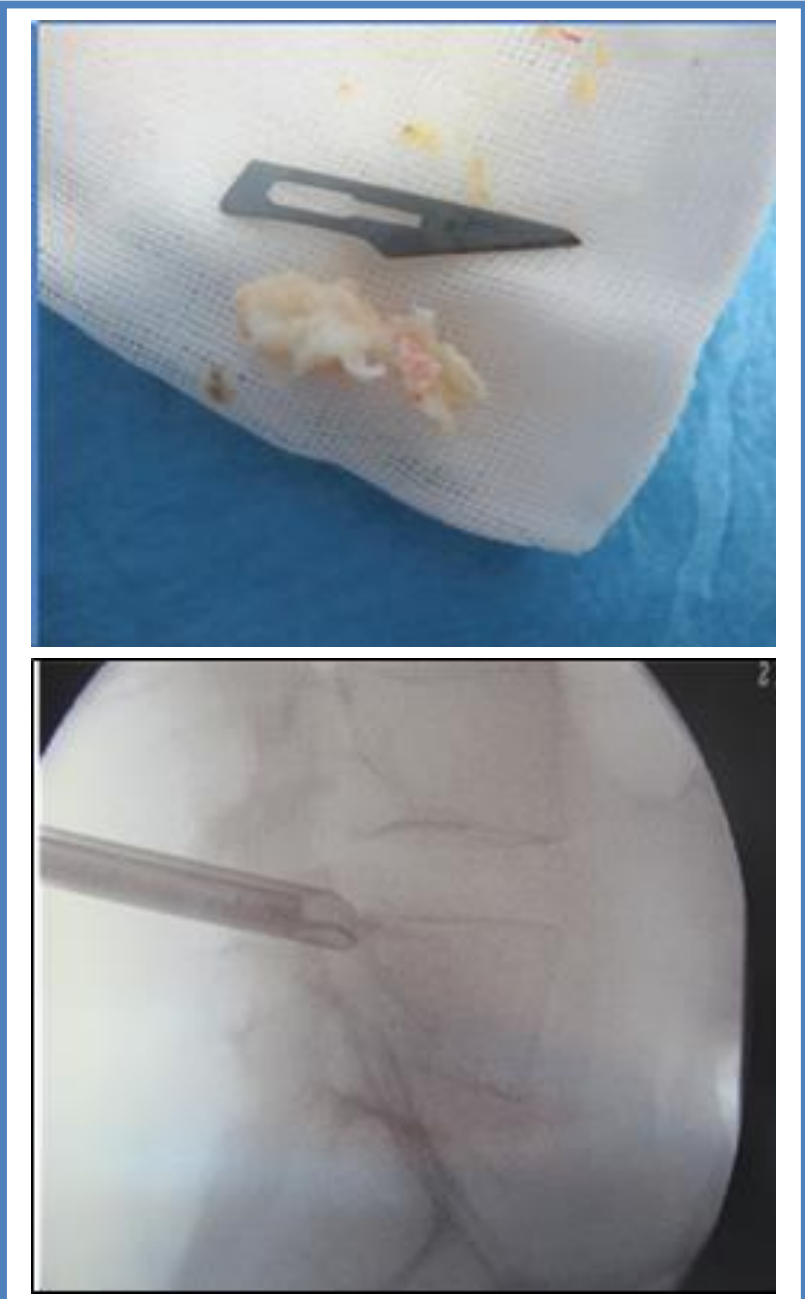

Fig 4. Intraoperative fluoroscopic view showing the working sleeve position with an extracted herniated disc.

an interlaminar approach by endoscopic excision. The operation was done on the side of the disc protrusion. After placing the patient in the prone position on a radiolucent table and sterile draping, general anesthesia was applied. Based on axial magnetic resonance or computed tomography, the entry point for endoscope was measure and marked. By using intraoperative fluoroscopy (C-arm) over the lamina in the anteroposterior projection, we made an incision at the exact interlaminar space in fluoroscopy. Unscarred tissue skin was incised around $10 \mathrm{~mm}$ in length. A dilator $(\varnothing$. $6.9 \mathrm{~mm}$ ) was inserted directly over the ligamentum flavum, followed by immediate anteroposterior and lateral lumbar spine imaging to check the accurate placement of the dilator. Then, an 8 -mm working sleeve (Ø $8.0 \mathrm{~mm}$ ) with bevel tip [working sleeve with $30^{\circ}$ bevel, working length (WL) $185 \mathrm{~mm}$ ] was introduced over the dilator and placed close to the lateral edge of the interlaminar space and ligamentum flavum. At this point, an endoscope system was inserted. Under direct endoscopic vision and constant irrigation, once the 


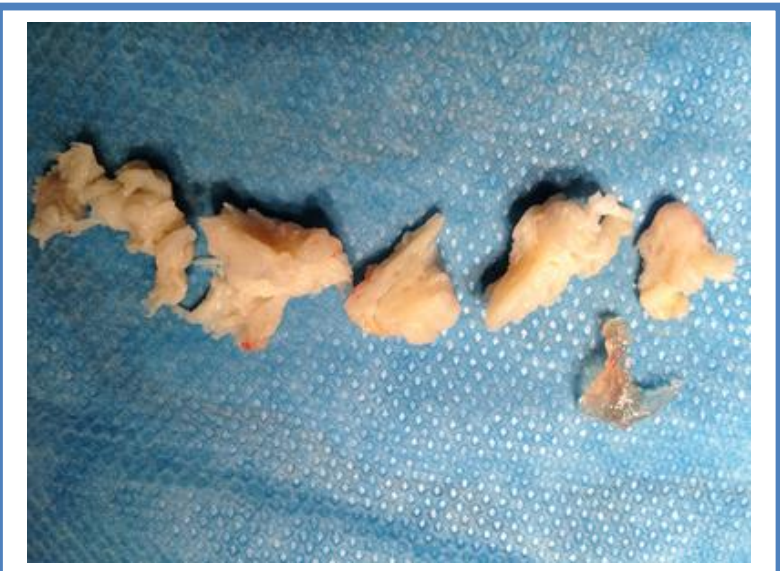

Fig 5. Multiple fragments of herniated disc and fragment of fibrin glue removed from inter laminar approach after recurrence

ligamentum flavum has been opened the cutter were entered. In case of canal stenosis or narrowed interlaminar space, minimal laminectomy and/or minimal facetectomy were precisely done via micro punch (Ø $2.5 \mathrm{~mm}$, WL $360 \mathrm{~mm}$ ) and/or oval burr, laterally hooded ( $\varnothing 4.0 \mathrm{~mm}$, WL $350 \mathrm{~mm})$ to afford a good view of the dura mater (Figure 2). It is worth mentioning that the dura mater was highly protected by the $30^{\circ}$ beveled working sleeve and the laterally hooded oval burr. Unraveling the herniated disc was feasible by putting aside the dura mater with aid of an atraumatic dissector (Ø $2.5 \mathrm{~mm}$, WL $350 \mathrm{~mm}$ ). The dura was displaced, and by rotating the working sleeve a safe window for nucleotomy was created (Figure 3, upper panel). The proper amount of nucleus pulposus was extracted by Micro-rongeur with long jaws (Ø $2.5 \mathrm{~mm}$, WL $360 \mathrm{~mm}$ ) (Figure 3, Lower panel). The homeostasis and shrinkage of annulus fibrosus was accelerated by annuloplasty via radiofrequency trigger $(450 \mathrm{MHz})$ flex bipolar electrodes (Surgitron Unit, Ellman, NY, USA). After generating a good view of the operative field (Figure 2,4), we selected patients who could receive sealants. Patients in grade 3 were considered the best candidates for injecting fibrin sealants. In lesions larger than $5 \mathrm{~mm}$, Fibrin sealants (Tisseel VH, Baxter Healthcare Corp., Deerfield, IL, USA) containing sealer protein concentrates, fibrinolysis inhibitor solution, thrombin, and calcium chloride solution were injected via endoscope slowly by a double-syringe delivery. At the conclusion of the surgery, patients in the control group received an epidural injection of a steroid (1\% prednisolone acetate). Eventually, the skin was closed by an intra-cutaneous-Silk stitch.

\section{Statistical methods}

The mean \pm standard deviation or median (min-max) for continuous variables and frequency $(\%)$ for categorical variables is reported as descriptive statistics. Continuous variables were compared in the study groups using

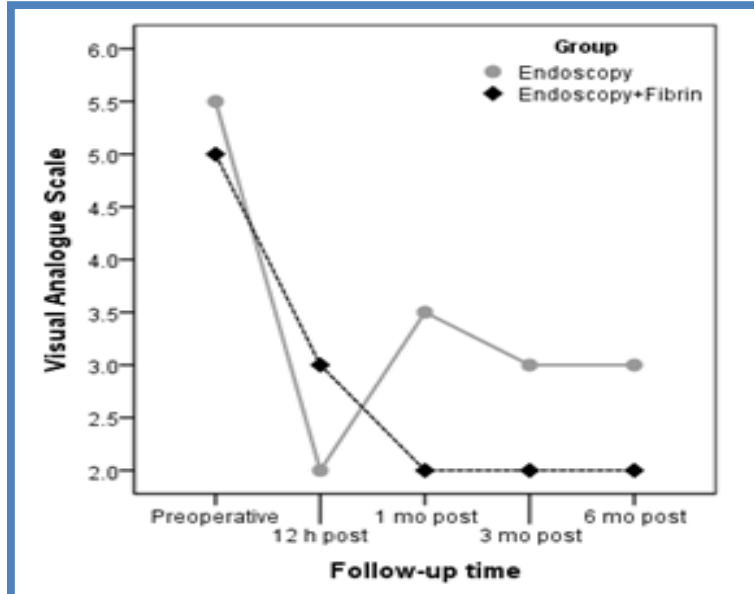

Fig. 6. Median VAS score changes over the follow-up time in both groups.

independent t-tests or the Mann-Whitney U test. The chisquared test or Fisher's exact test were used to compare the categorical variables between groups. $P$ values less than 0.05 were considered statistically significant. SPSS software (SPSS Statistics for Windows, Version 22.0. IBM Corp., Armonk, NY) was used for all statistical analyses.

\section{Results}

A total of 45 patients were enrolled in this study. Ten patients who missed follow-up appointments, for several reasons, were excluded from our analysis. Clinical and imaging follow-up data were obtained for all 35 patients (28 men, 7 women) with an average age of 53.09 \pm 10.82 , including 18 patients who underwent endoscopic discectomy and 17 patients who underwent endoscopic discectomy plus fibrin sealant (Figure 5). Baseline patient demographics are presented in Table 1. The mean patient age in the endoscopic discectomy group was $55.39 \pm 11.26$ years compared to $50.65 \pm 10.09$ years in the endoscopic discectomy plus fibrin sealant group $(P=0.20)$. The main sex preponderance of each group was men, with 16 men $(88.9 \%)$ in the endoscopic discectomy group and 12 men $(70.6 \%)$ in the endoscopic discectomy plus fibrin sealant group. Of the enrolled patients, $71.42 \%$ had lower back pain for more than 6 months. Median size of annular tearing was significantly lower in the endoscopic discectomy group (median, 3) (minimum, 2; maximum, 5); however, the corresponding factor in the endoscopic discectomy plus fibrin sealant group was significantly larger (median, 6) (minimum, 5; maximum, $10)$, with $P<0.001$. During routine follow-up, the VAS score markedly decreased in both groups 12 hours after endoscopic treatment compared to the VAS score before treatment. The median VAS score was significantly lower in the endoscopic discectomy plus fibrin sealant group than in the endoscopic discectomy group 1-3 months after endoscopic treatment $(P=0.002$ and $P=$ 0.015 , respectively) (Figure 6). Furthermore, the median 
Fibrin sealants to prevent recurrent lumbar disc herniation

Eur J Transl Myol 30 (2): 325-333, 2020

Table 1. Characteristics of 35 patients which classified into two groups.

\begin{tabular}{ccccc}
\hline Variable & & A & B & P-value \\
\hline Age (year) & & $55.39 \pm 11.26$ & $50.65 \pm 10.09$ & 0.20 \\
Sex & Male & $16(88.9)$ & $12(70.6)$ & 0.23 \\
& Female & $2(11.1)$ & $5(29.4)$ & \\
& & & & \\
& No & 13 & 10 & 0.13 \\
Atrophy before operation & Yes & 5 & $6.00(4-10)$ & 0.37 \\
Duration of symptom & & $6.25(3-18)$ & & \\
(months) & & $4.25(3-7)$ & $5.00(3-7)$ & \\
Lesion size & & & & \\
\hline
\end{tabular}

Data are represented as mean \pm standard deviation, median (minimum and maximum), or frequency (percentage). A: Endoscopic discectomy; B: Endoscopic discectomy + fibrin sealants

degree of Lasègue's sign was diminished after endoscopic treatment in both groups. Median degree of Lasègue's sign 6 months after treatment in the endoscopic discectomy group was $30^{\circ}$. Conversely, in the endoscopic discectomy plus fibrin sealant group, the median degree of Lasègue's sign 6 months after treatment was $20^{\circ}(P=0.02)$ (Table 2). Reflex tests showed that it took nearly 6 months for patients in both groups to regain approximately intact reflexes (16 intact reflexes in the endoscopic discectomy group vs. 15 intact reflexes in the endoscopic discectomy plus fibrin sealant group) $(P=0.99)$ (Table 3). There was no significant difference between the recurrence rate of HNP between the two groups.

Only one patient in the endoscopic discectomy group had HNP recurrence during follow-up compared to two patients in the endoscopic discectomy plus fibrin sealant group. Owing to rare cases of recurrence and the small

Table 2. Changes of VAS score, Lasègue's sign and Muscle strength during routinely follow-up in both groups.

\begin{tabular}{lccc}
\hline Variable & A & B & P-value \\
\hline VAS Score & & $5(3-9)$ & 0.59 \\
Preoperative & $5.5(4-8)$ & $3(1-4)$ & 0.27 \\
12 hours & $2(1-4)$ & $2(1-3)$ & $0.002^{*}$ \\
1 month & $3.5(2-5)$ & $2(2-4)$ & $0.015^{*}$ \\
6 months & $3(2-5)$ & & \\
Lasègue's Sign & & $45(30-80)$ & 0.91 \\
Preoperative & $45(30-70)$ & $30(20-60)$ & 0.88 \\
1 month & $30(20-50)$ & $20(15-70)$ & $0.02^{*}$ \\
6 months & $30(20-45)$ & & 0.71 \\
Muscle Strength & & $5(3-5)$ & 0.48 \\
\hline Preoperative & $5(3-5)$ & $4(2-5)$ & \\
6 months & $4.5(3-5)$ & & \\
\hline
\end{tabular}

Data are represented as median (minimum and maximum). VAS: Visual Analogue Scale. A: Endoscopic discectomy; $B$ : Endoscopic discectomy + fibrin sealants. *Statistically significant difference 
Fibrin sealants to prevent recurrent lumbar disc herniation

Eur J Transl Myol 30 (2): 325-333, 2020

Table 3. Reflex changes in both groups: before operation, 1 month and 6 months after operation respectively.

\begin{tabular}{|c|c|c|c|}
\hline Reflex & $\mathbf{A}$ & B & P-value \\
\hline Preoperative & & & $0.018^{*}$ \\
\hline Alleviated & 0 & 0 & \\
\hline Impaired & 4 & 11 & \\
\hline Intact & 14 & 6 & \\
\hline 1 month & & & 0.12 \\
\hline Alleviated & 1 & 2 & \\
\hline Impaired & 4 & 9 & \\
\hline Intact & 13 & 6 & \\
\hline 6 months & & & 0.99 \\
\hline Alleviated & 1 & 1 & \\
\hline Impaired & 1 & 1 & \\
\hline Intact & 16 & 15 & \\
\hline
\end{tabular}

A: Endoscopic discectomy; B: Endoscopic discectomy + fibrin sealants. ${ }^{*}$ Statistically significant difference.

group study, no statistical analysis method was available for distinguishing recurrence between two groups.

\section{Discussion}

In our study, we compared endoscopic lumbar discectomy with endoscopic disc surgery in conjunction with fibrin sealant. Discogenic LBP is seen in $25-45 \%$ of patients. $^{2,10}$ Long-lasting and recurrent pain can be associated with herniated discs. Stability of spinal disc materials as a part of the shock absorber system may be deteriorated by vigorous, long-standing activity, leading to bulging, protruding, extruding, or sequestration of disc material in intervertebral or vertebral foramina. Release of inflammatory compounds such as interleukin (IL-1 $\alpha / \beta$, IL-6, IL-8, IL-17), and tumor necrosis factor- $\alpha$ as a consequence of nucleus pulposus derangement and extracellular matrix breakdown will amplify the inflammatory cascade via activating macrophage migration. Nerve damage can ensue from a slipped disc after days to weeks by putting pressure on the spinal nerves. Inducing pain transmission can be enhanced by increased painful neuropeptides such as substance $\mathrm{P}$ and calcitonin gene-related peptide, known potential factors for pain generation. ${ }^{11-14}$ Common manifestations of slipped discs in patients can be varied based on the size and position of the herniated disc, but they can include ipsilateral pain and numbness, unexplained muscle weakness, and pain in the buttocks, thigh, or calf. Neural compression and subsequent spinal canal narrowing are other major contributing factors for patient symptoms. ${ }^{15,16}$ By developing advanced endoscopic discectomy as a type of tissue-preserving surgery, treatment of patients with HNP has gained more attention. Recently, fibrin glue as an exemplary substance for bone tissue engineering applications has received attention. In 1940, Tidrick et al. introduced fibrin sealant as a promising agent for efficient hemostasis and safe dural closure in skin graft fixations ${ }^{17}$. Its use is widely accepted in several specialties. In fact, Panda et al. demonstrated fibrin sealants as a type of tissue adhesive for conjunctival closure after pterygium, strabismus surgery, ${ }^{18}$ and for fracture fixation of osteochondral fragments in a study by Kirilak Y et al. ${ }^{19}$ This blood-derived product is composed of two components in distinct vials: a freeze-dried concentrate of clotting proteins, mainly fibrinogen, factor XIII, and fibrin in the first vial and a freeze-dried coagulative serine protease called thrombin in the second vial. ${ }^{20}$ Freeze-dried fibrin particle of fibrin glue with adhesive properties can accelerate the final steps of coagulation. Triggering coagulation cascade, activating thrombin, stabilizing clots, and promoting polymerization have been discussed as a possible role of thrombin in homeostasis promotion. ${ }^{21}$ Owing to the autologous characteristics of fibrin sealant, the possibility of being encountered with bovine proteins is eliminated and human-born contamination is negligible, demonstrating its good biocompatibility and biodegradability. ${ }^{22}$ Fibrin glue has been proposed as a scaffold for orchestrating fibroblasts, stimulating mesenchymal stem cells and promoting angiogenesis by its inherent extracellular matrix proteins such as fibronectin, vascular endothelial growth factor, and plasminogen. ${ }^{20,23}$ This promising autologous sealant, with its osteogenic, osteoinductive, and osteoconductive characteristics, ${ }^{24}$ enhances bone regeneration and bone healing by improving the 
remodeling process, mainly secondary to its factor XIII, which accelerates bone healing through the development of fibrin cross linking, fibroblasts, and undifferentiated mesenchymal cells. ${ }^{25-30}$ Dural closure and preventing local major spinal hemorrhage are the other two primary benefits. In combination with these features, it is clear that fibrin sealant can be a good choice to fulfill our requirement in providing scaffold and preventing recurrence of disc herniation.

Previously, a system was suggested for depicting the size and location of disc herniation based on MRI findings. In 1997, McCulloch et al. proposed lumbar disc herniation classification classification named as Michigan State University (MSU) based on disc location and spinal canal structure. ${ }^{31}$ MSU classification has gained popularity as a precise noninvasive preoperative evaluation factor determined by surgeons. However, we have proposed our classification under direct visualization to include eligible patients for fibrin sealant, coupled with preoperative MRI for more accurate evaluation and strategy selection. We think that patients with disc herniation larger than $5 \mathrm{~mm}$ will benefit more in terms of diminished incidence of recurrence over the duration of follow-up in endoscopic discectomy with fibrin sealant group. In the late 1980s, spinal endoscopy was performed by extracting the main offender of increased intradiscal pressure. ${ }^{32,33}$ Spinal endoscopy gains momentum due to recent technical advances in video processing equipment, numerous specialized spinal instruments, and early postoperative rehabilitation. Until now, the superiority of this technique over other conservative therapies is a moot point. ${ }^{34}$ Reduced surgical complications were reported in endoscopic disc surgery. ${ }^{35,36}$ Ruetten et al. demonstrated faster return to work with shorter hospital stays, reduced operating time, and reduced scarring of the skin tissue with endoscopic technique compared to conventional surgery. There is no need to remove bone and muscle to access to herniated discs, and the probability of scar adhesion after endoscopy is negligible. ${ }^{37}$ In people between 25 and 55 years old, nearly $95 \%$ of herniated nucleus pulposus occurs at L5-S1, ${ }^{38}$ reaffirming the high prevalence of L5-S1 disc involvement in our data. Location, type, and degree of disc herniation should be evaluated, to determine the interlaminar type of endoscopic disc surgery or the transforaminal approach. Given the anatomic features of L5-S1, high iliac crest, and small size of foramen, some studies gave precedence to the interlaminar approach over the transforaminal approach in the axillary type of disc herniation and migrated discs. ${ }^{38,39}$ By performing the interlaminar technique, enough visual field would be provided compared to the anatomical barrier in the transforaminal approach. These methods are common practice in orthopedic surgery. ${ }^{40-43}$ The interlaminar approach provides direct access to the herniation associated with potential risk of dura mater rupturing. However, factoring in all the advantages and disadvantages, based on our experience, we recommend the interlaminar approach to easily access the herniation. We did not find enough evidence to support any significant difference in outcomes between endoscopic discectomy surgery and endoscopic disc surgery accompanied by fibrin sealant. After comparing the recurrence rate of these two endoscopic surgery techniques, the re-herniation and reoperation rates between our study and the control group were insignificant. We hypothesize that the recurrence rate is highly attributed to the presence of high-grade size of annular tearing, which was more prevalent in the study group. Somehow, the recurrence rate could be decreased if we included low-grade tear individuals in them. Due to the temporary effects of fibrin sealant in preventing disc herniation in our study and insignificant HNP recurrence rate in both the study and control groups, we assume that by using fibrin sealant, early HNP recurrence (less than 6 months) can be prevented.

This study had some limitations: Firstly, the analyzed recordings came from a relatively small number of patients, with no control group for herniations larger than $5 \mathrm{~mm}$ and no low to moderate-grade tear in study group. Secondly, this was an observational cross-sectional study, which means that it has less power than randomized and blinded research. Larger multicenter studies with long-term follow-up should be performed to precisely assess this technique.

In conclusion, owing to the temporary effects of fibrin sealant in preventing disc herniation and the nearly same observed recurrence rate in both the case and control groups, the results of this study suggest the role of fibrin sealants combined with endoscopic discectomy to prevent early HNP recurrence rate.

\section{List of acronyms}

HNP- herniated nucleus pulposus

LBP- Low back pain

MRI- magnetic resonance imaging

MSU - Michigan State University

VAS - Visual Analogue Scale

WL - Working length

\section{Authors contributions}

All authors played a substantial role in conception and/or study design, drafting and finalizing of the manuscript. They agree for all aspects of accuracy and integrity of the work.

\section{Acknowledgments None}

Funding No funding was obtained for this project.

\section{Conflict of Interest}

The authors declare they have no financial, personal, or other conflicts of interest.

\section{Ethical Publication Statement}

We confirm that we have read the Journal's position on issues involved in ethical publication and affirm that this report is consistent with those guidelines. 


\section{Corresponding Author}

Shahram Akhlaghpoor, MD. Associate Professor of Radiology. Address: Pardis Noor Medical Imaging Center, No 5, 25 ${ }^{\text {th }}$ street, Sa'adat abad street, Tehran, Iran. Tel: 09121096762 / +98-21-22258248;

ORCID iD: 0000-0001-9993-3801

Email:shahram_ak@yahoo.com

\section{Emails of coauthors}

Pooya Torkian: Pooyaaig@yahoo.com ORCID iD: 0000-0001-6147-8145

Keivan Daneshvar: Keivan.Daneshvar@insel.ch ORCID iD: 0000-0002-0345-0379

Ebrahim Taherian: Karimi1384@yahoo.com ORCID iD: 0000-0003-4942-4528

Yasaman Rezaeifar: Yasi_rezaeifar@yahoo.com ORCID iD: 0000-0001-6535-5062

\section{References}

1. Weber H. Lumbar disc herniation: a controlled, prospective study with ten years of observation. Spine 1983;8:131-40.

2. Deyo RA, Tsui-Wu Y-J. Descriptive epidemiology of low-back pain and its related medical care in the United States. Spine 1987;12:264-8.

3. Ahn Y, Lee SH. Outcome predictors of percutaneous endoscopic lumbar discectomy and thermal annuloplasty for discogenic low back pain. Acta Neurochir (Wien) 2010;152:1695-702. doi: 10.1007/s00701-010-0726-2. Epub 2010 Jul 7.

4. Choy DS. Percutaneous laser disc decompression (PLDD): twelve years' experience with 752 procedures in 518 patients. J Clin Laser Med Surg $1998 ; 16: 325-31$.

5. Aizawa T, Ozawa H, Kusakabe T, et al. Reoperation rates after fenestration for lumbar spinal canal stenosis: a 20-year period survival function method analysis. Eur Spine J 2015;24:381-7. doi: 10.1007/s00586-014-3479-4. Epub 2014 Jul 30.

6. Tsou PM, Yeung CA, Yeung AT. Posterolateral transforaminal selective endoscopic discectomy and thermal annuloplasty for chronic lumbar discogenic pain: a minimal access visualized intradiscal surgical procedure. Spine J 2004;4:56473.

7. Lee S-H, Kang HS. Percutaneous endoscopic laser annuloplasty for discogenic low back pain. World Neurosurg 2010;73:198-206; discussion e33. doi: 10.1016/j.surneu.2009.01.023. Epub 2009 Mar 27.

8. Esposito F, Angileri FF, Kruse P, et al. Fibrin sealants in dura sealing: a systematic literature review. PLoS One 2016;11:e0151533. doi: 10.1371/journal.pone.0151533. eCollection 2016.

9. Epstein NE. A review article on the benefits of early mobilization following spinal surgery and other medical/surgical procedures. Surg Neurol Int
2014;5:S66-73. doi: 10.4103/2152-7806. 130674. eCollection 2014.

10. Hoy D, Brooks P, Blyth F, et al. The epidemiology of low back pain. Best Pract Res Clin Rheumatol 2010;24:769-81. doi: 10.1016 /j.berh.2010.10.002.

11. Medzhitov R. Origin and physiological roles of inflammation. Nature 2008 ;454:428-35. doi: 10.1038/nature0720.

12. Molinos M, Almeida CR, Caldeira J, et al. Inflammation in intervertebral disc degeneration and regeneration. J Royal Soc Interface. 2015;12:20141191.

13. Risbud MV, Shapiro IM. Role of cytokines in intervertebral disc degeneration: pain and disc content. Nat Rev Rheumatol. 2014;10:44-56. doi: 10.1038/nrrheum.2013.160. Epub 2013 Oct 29.

14. Takahashi H, Suguro T, Okazima Y, et al. Inflammatory cytokines in the herniated disc of the lumbar spine. Spine 1996;21:218-24.

15. Peterson CK, Pfirrmann CW, Hodler J, et al. Symptomatic, Magnetic Resonance ImagingConfirmed Cervical Disk Herniation Patients: A Comparative-Effectiveness Prospective Observational Study of 2 Age-and Sex-Matched Cohorts Treated With Either Imaging-Guided Indirect Cervical Nerve Root Injections or Spinal Manipulative Therapy. J Manipulative Physiol Ther 2016;39:210-7. doi: 10.1016/ j.jmpt.2016.02.004. Epub 2016 Mar 31.

16. Lee JH, Lee S-H. Clinical and radiological characteristics of lumbosacral lateral disc herniation in comparison with those of medial disc herniation. Medicine (Baltimore) 2016;95:e2733. doi: 10.1097/MD.0000000000 002733.

17. Tidrick R, Warner E. Fibrin fixation of skin transplants. Surg 1944;15:90-5.

18. Panda A, Kumar S, Kumar A, et al. Fibrin glue in ophthalmology. Indian J Ophthalmol 2009;57:3719. doi: 10.4103/0301-4738.55079.

19. Kirilak Y, Pavlos NJ, Willers CR, et al. Fibrin sealant promotes migration and proliferation of human articular chondrocytes: possible involvement of thrombin and protease-activated receptors. Int J Mol Med 2006;17:551-8.

20. Dickneite $\mathrm{G}$, Metzner $\mathrm{H}$, Pfeifer $\mathrm{T}$, et al. A comparison of fibrin sealants in relation to their in vitro and in vivo properties. Thromb Res 2003;112:73-82.

21. Thompson DF, Letassy NA, Thompson GD. Fibrin glue: a review of its preparation, efficacy, and adverse effects as a topical hemostat. Drug Intell Clin Pharm 1988;22:946-52.

22. Isogai N, Landis WJ, Mori R, et al. Experimental use of fibrin glue to induce site-directed osteogenesis from cultured periosteal cells. Plast Reconstr Surg 2000 ;105:953-63. 
23. Polo-Corrales L, Latorre-Esteves M, Ramirez-Vick JE. Scaffold design for bone regeneration. J Nanosci Nanotechnol 2014;14:15-56.

24. Moore WR, Graves SE, Bain GI. Synthetic bone graft substitutes. ANZ J Surg 2001;71:354-61. Review.

25. Lee O, Coathup M, Goodship A, et al. Use of mesenchymal stem cells to facilitate bone regeneration in normal and chemotherapy-treated rats. Tissue Eng 2005;11:1727-35.

26. Abiraman S, Varma H, Umashankar P, et al. Fibrin glue as an osteoinductive protein in a mouse model. Biomaterials 2002;23:3023-31.

27. Tayapongsak P, O'Brien DA, Monteiro CB, et al. Autologous fibrin adhesive in mandibular reconstruction with particulate cancellous bone and marrow. J Oral Maxillofac Surg 1994;52:161-5; discussion 166.

28. Class L, Burri C, Gerngross H, et al. Bone healing stimulated by plasma Factor XIII: Osteotomy experiments in sheep. Acta Orthop Scand 1985;56:57-62.

29. Ito K, Yamada Y, Naiki T, et al. Simultaneous implant placement and bone regeneration around dental implants using tissue-engineered bone with fibrin glue, mesenchymal stem cells and plateletrich plasma. Clin Oral Implants Res 2006 ; 17:57986.

30. Giannini G, Mauro V, Agostino T, et al. Use of autologous fibrin-platelet glue and bone fragments in maxillofacial surgery. Transfus Apher Sci 2004;30:139-44.

31. Wiltse LL, Berger PE, McCulloch JA. A system for reporting the size and location of lesions in the spine. Spine 1997;22:1534-7.

32. Kovac D. Automated endoscopic percutaneous diskectomy in the treatment of lumbar disk hernia. Lijec Vjesn 1991;113:158-61.

33. Mayer H, Brock M, Berlien HP, et al. Percutaneous Endoscopic Laser Discectomy (PELD) A New Surgical Technique for Non-sequestrated Lumbar Discs. Acta Neurochir Suppl (Wien) 1992;54:53-8.

34. Birkenmaier C, Komp M, Leu HF, et al. The current state of endoscopic disc surgery: review of controlled studies comparing full-endoscopic procedures for disc herniations to standard procedures. Pain Physician 2013;16:335-44.

35. Ruetten S, Komp M, Merk H, et al. Full-endoscopic anterior decompression versus conventional anterior decompression and fusion in cervical disc herniations. Int Orthop 2009;33:1677-82. doi: 10.1007/s00264-008-0684-y. Epub 2008 Nov 18.

36. Ruetten S, Komp M, Merk H, et al. Full-endoscopic interlaminar and transforaminal lumbar discectomy versus conventional microsurgical technique: a prospective, randomized, controlled study. Spine (Phila Pa 1976). 2008;33:931-9. doi: 10.1097/BRS.0b013 e31816c8af7.

37. Hou T, Zhou Q, Dai F, et al. Repeated microendoscopic discectomy for recurrent lumbar disk herniation. Clinics (Sao Paulo) 2015;70:120-5. doi: 10.6061/clinics/2015(02) 09.

38. Jordan JL, Konstantinou K, O'Dowd J. Herniated lumbar disc. BMJ Clin Evid 2011;2011. pii: 1118. Review.

39. Choi G, Lee SH, Raiturker PP, et al. Percutaneous endoscopic interlaminar discectomy for intracanalicular disc herniations at L5-S1 using a rigid working channel endoscope. Neurosurgery 2006 ;58:ONS59-68; discussion ONS59-68.

40. Moghtadaei M, Otoukesh B, Bodduhi B, et al. Evaluation of Patellar Position before and After Medial Opening Wedge High Tibial Osteotomy: Radiographic and Computed Tomography Findings. Med Arch. 2016;70:293-295.

41. Yeganeh A, Otoukesh B, Kaghazian P, et al. Evaluation of the Etiologies of Implant Fracture in Patients With Fractures of the Implants of Lower Limbs' Long Bones. Med Arch. 2015;69:405-408.

42. Yeganeh A, Taghavi R, Moghtadaei M. Comparing the Intramedullary Nailing Method Versus Dynamic Hip Screw in Treatment of Unstable Intertrochanteric Fractures. Med Arch. 2016;70:5356.

43. Yeganeh A, Alaee A, Boddouhi B, et al. Results of surgically treated talar fractures. Chin J Traumatol. 2013;16:361-364.

Submitted: December 10, 2019

Revision received: January 24, 2020 Accepted for publication: January 24, 2020 